\title{
ECUACIÓN DE LA ONDA PLANA EN UN ESPACIO NO-ARQUIMEDIANO CON AMORTECIMIENTO
}

\section{EQUATION OF THE FLAT WAVE IN A NON- ARCHIMEDIAN SPACE WITH DECAY}

\author{
(iD) Alex Armando Cruz Huallpara ${ }^{1,2}$ \\ 'Universidad Nacional Mayor de San Marcos. Lima' Perú \\ Universidad Nacional del Santa’ Ancash. Lima· Perú \\ Correspondencia: \\ Dr. Alex Armando Cruz Huallpara \\ acruzh@unmsm.edu.pe \\ 2018825004@uns.edu.pe
}

\section{RESUMEN}

El presente artículo, considera K un cuerpo local no- arquimediano, se muestra que para solucionar la ecuación de la onda plana sobre un espacio no arquimediano se considera una función de prueba $f\left(t+w_{1} x_{1}+w_{2} x_{2}+\cdots+w_{n} x_{n}\right)$ de valor complejo Bruhat - Schwartz en $\mathrm{K}_{1}\left(t, x_{1}, x_{2}, \cdots, x_{n}\right) \in K^{n+1}, \max _{1 \leq j \leq n}\left|w_{i}\right|=1$, que satisface para algún $f$, para un cierta ecuación homogénea pseudo-diferenciaal, un análogo a la ecuación de la onda clásica, se desarrolla la teoría del problema de Cauchy para la ecuación de la onda plana sobre espacio no arquimediano.

Palabras clave: función de prueba, espacio no-arquinediano, pseudo-diferencial, ecuación de la onda.

\section{ABSTRACT}

This article $\mathrm{K}$ be considered a non-Archimedean local body, it is shown that to solve the equation of the plane wave over a non-Archimedean space we consider a test function $f\left(t+w_{1} x_{1}+w_{2} x_{2}+\cdots+w_{n} x_{n}\right)$ of complex value Bruhat - Schwartz in $\mathrm{K}$, $\left(t, x_{1}, x_{2}, \cdots, x_{n}\right) \in K^{n+1}, \max _{1 \leq j \leq n}\left|w_{i}\right|=1$, which satisfies for some $f$, for a certain homogeneous pseudo-differential equation, an analog to the classical wave equation, the theory of the Cauchy problem is developed for the equation of the plane wave over non-Archimedean space.

Key words: test function, non-Archinedian space, pseudo-differential, wave equation. 


\section{INTRODUCCIÓN}

La investigación realizada referente a la solución de la ecuación de la onda plana no-arquimediano con amortecimiento. Para solucionar la ecuación debemos conocer las ecuaciones pseudo-diferenciales para funciones de valores complejos definidas en campos locales no arquimedianos, en particular el campo $Q p$ de números p-ádicos, son cada vez más importantes.

En esta investigación se propone un enfoque alternativo; En lugar de una semejanza formal en la definición de una ecuación, se parte de sus propiedades. Llamemos a una función $u\left(t, X_{1}, \ldots, X_{n}\right): Q_{p}{ }^{n} \rightarrow C$ una onda plana, si:

$$
u\left(t, x_{1}, \ldots, x_{n}\right)=f\left(t+w_{1} x_{1}+\ldots+w_{n} x_{n}\right)
$$

donde $f$ pertenece al espacio de Bruhat-Schwartz $\mathrm{D}\left(Q_{p}\right)$ de funciones de prueba

$$
w_{1}, \ldots, w_{n} \in Q_{p}, \max _{1 \leq j \leq n}\left|w_{j}\right|_{p}=1
$$

Demostraremos que toda función (1) es una solución de la ecuación:

$$
D_{t}^{\alpha} u-D_{x}^{\alpha, n} u=0
$$

donde $D^{\alpha}$ es el operador de diferenciación fraccional de Vladimirov (2003), es decir, un operador pseudo-diferencial con el símbolo $|\xi|_{p}^{\alpha}$, mientras que $D_{x}^{\alpha, n}$ es un operador pseudo-diferencial de $\mathrm{n}$ variables con el símbolo $\max _{1 \leq j \leq n}\left|\xi_{j}\right|_{p}^{\alpha}, \alpha>0$ es un número arbitrario.

Considere la ecuación para una solución fundamental relacionada E:

$$
D_{t}^{\alpha} E-D_{x}^{\alpha, n} E=\delta
$$

donde $E$ pertenece a alguna clase de distribuciones, en las que se definen los operadores, con las relaciones habituales entre ellos y la transformada de Fourier. Entonces, realizando la transformada de Fourier obtenemos la identidad contradictoria:

$$
\left(|\tau|_{p}^{\alpha}-\max _{1 \leq j \leq n}\left|\xi_{j}\right|_{p}^{\alpha}\right) \tilde{E}\left(\tau, \xi_{1}, \xi_{2}, \ldots \xi_{n}\right)=1
$$

donde el lado izquierdo desaparece en el conjunto abierto:

$$
\left\{0 \neq\left(\tau, \xi_{1}, \xi_{2}, \ldots \xi_{n}\right) \in Q_{p}^{n+1}:|\tau|_{p}=\max _{1 \leq j \leq n}\left|\xi_{j}\right|_{p}^{\alpha}\right\}
$$

Por lo tanto, la solución fundamental no puede existir y no se puede esperar ningún comportamiento razonable de una ecuación no homogénea asociada con (2).

Sin embargo, en este trabajo probamos la existencia y unicidad de soluciones para algunos análogos del problema de Cauchy para la ecuación (2) en la clase de funciones radiales. 


\section{MATERIAL Y METODOS}

Se utilizó el método científico, la investigación es de nivel analítico, debido a que se utiliza la teoría de pseudo diferencial, para solucionar la ecuación de la onda plana en un espacio no arquimediano.

Sea $K$ un cuerpo local no arquimedeano, que es un campo topológico local no compacto totalmente disconexo y no discreto. Es bien sabido que $K$ es isomorfo a una extensión finita del cuerpo $K$ de los números p-adic (si $K$ tiene la característica 0), o al cuerpo de las series formales de Laurent con coeficientes de un cuerpo finito $F_{q}$, si $K$ tiene la característica $p \neq 0$; en este caso $q=p^{v}, v \in N$. Para un resumen de las principales nociones y resultados con respecto a los cuerpos locales.

Cualquier cuerpo local está dotado de un valor absoluto $|\cdot|$, tal que:

i) $\quad|x|=0$ sí $y$ solo si $x=0$

ii) $\quad|x y|=|x| \cdot|" y "|$

iii) $\quad|x+y| \leq \max (|x|,|y|)$

Denote $0 "="\{x \in K:|x| \leq 1\}, P=\{x \in K:|x|<1\}, U=0 \backslash P$. O es una subanilo de $K$ llamada anillo de enteros, $\mathrm{P}$ es un ideal en $\mathrm{O}$ llamado ideal primo; el grupo multiplicativo $U$ se llama grupo de unidad. $\mathrm{El}$ ideal $\mathrm{P}$ contiene un elemento $\beta$ tal que $\mathrm{P}=\beta \mathrm{O}$ (un elemento primo). El anillo de cociente $\mathrm{O} \backslash \mathrm{P}$ es en realidad un cuerpo finito; denotan por q su cardinalidad. Siempre asumiremos que el valor absoluto está normalizado, es decir $|\beta|=\mathrm{q}^{-1}$. El valor absoluto normalizado $|\cdot|$ toma los valores $\mathrm{q}^{\mathrm{N}}$, $N \in Z$.

$\mathrm{Si} K=\mathrm{Q}_{\mathrm{p}}$ ( $\mathrm{p}$ es un número primo), que es una completitud del cuerpo $\mathrm{Q}$ de números racionales con respecto al valor absoluto: $|x|_{p}=p^{-v}$ para $x=p^{v} \cdot\left(\frac{m}{n}\right)$

Donde $v, m, n \in Z$, y $m, n$ son primos a $p$, entonces $\beta=p$ ( $p$ es visto como un elemento de $Q \_p$ ) y $q=p$ (como un número natural).

Volviendo a un cuerpo local general $\mathrm{K}$, denote por $S \subset O$ un sistema completo de representantes de las clases de residuos de $\mathrm{O} / \mathrm{P}$. Entonces, cualquier elemento distinto de cero $x \in K$ admite la representación canónica en forma de serie convergente:

$$
x=\beta^{-n}\left(x_{0}+x_{1} \beta+x_{2} \beta^{2}+\cdots\right)
$$

Donde $n \in Z,|x|=q^{n}, x_{j} \in S, x_{0} \notin P$. Para $K=Q_{p}$, uno puede tomar $\mathrm{S}=\{0,1, \cdots, p-1\}$.

El grupo aditivo de cualquier cuerpo local es auto-dual, que es si $\chi$ es un carácter aditivo de valor complejo no constante de $\mathrm{K}$, cualquier otro carácter aditivo puede escribirse como $\chi_{a}(x)=\chi(a x)$ , $x \in K$, para algunos $a \in K$. Ver [7] para una descripción explícita del carácter utilizado en análisis armónico en cuerpos locales ("el carácter aditivo canónico"). En particular, se supone que es un carácter de rango cero, es decir $\chi(x) \equiv 1$ para $x \in O$, mientras que existe tal elemento $x_{0} \in K$ que $\mid x_{0}$ $\mathrm{I}=q y \chi\left(\mathrm{x}_{0}\right) \neq 1$.

La dualidad anterior se usa en la definición de la transformada de Fourier sobre K. Denotando por $\mathrm{dx}$ la medida de Haar en el grupo aditivo de $\mathrm{K}$ (normalizado de tal manera que la medida de $\mathrm{O}$ sea igual a 1) escribimos: $\tilde{f}(\xi)=\int_{K} \chi(x \xi) f(x) d x, \xi \in K$

donde fes una función de valor complejo de $L_{1}(K)$. Como de costumbre, la transformada de Fourier Fpuede extenderse de $L_{1}(K) \cap L_{2}(K)$ a un operador unitario en $L_{2}(K)$, Si $F f=f^{\sim} \in L_{1}(K)$, tenemos la fórmula de inversión:

$$
f(x)=\int_{K} \chi(-x \xi) \tilde{f}(\xi) d \xi
$$


Similar, si $f: K^{n} \rightarrow C$, Nosotros escribimos:

$$
\tilde{f}\left(\xi_{1}, \xi_{2}, \cdots, \xi_{n}\right)=\int_{K^{n}} \chi\left(x_{1} \xi_{1}+x_{2} \xi_{2}+\cdots+x_{n} \xi_{n}\right) f\left(x_{1}, x_{2}, \cdots, x_{n}\right) d x_{1} \ldots d x_{n}
$$

La fórmula de inversión es entonces:

$$
f\left(x_{1}, x_{2}, \cdots, x_{n}\right)=\int_{K^{n}} \chi\left(-x_{1} \xi_{1}-x_{2} \xi_{2}-\cdots-x_{n} \xi_{n}\right) \tilde{f}\left(\xi_{1}, \xi_{2}, \cdots, \xi_{n}\right) d \xi_{1} \ldots d \xi_{n}
$$

\section{Espacios de Funciones de Prueba y Distribuciones}

Una función $f: K \rightarrow C$ se llama localmente constante, si existe tal número entero l que para cualquier $x \in K$ :

$$
f\left(x+x^{\prime}\right)=f(x), \text { si }\left|x^{\prime}\right| \leq q^{-l}
$$

El número más pequeño $l$ con esta propiedad se llama exponente de la constancia local de la función $f$.

Denote con $D(K)$ el conjunto de todas las funciones constantes localmente con soportes compactos. Para proporcionarle una topología, considere un subespacio $D_{N}^{l}(K) \subset D(K)$ de funciones con soportes en la bola:

$$
B_{N}=\left\{x \in K ;|x| \leq q^{N}\right\}, \quad \mathrm{N} \in \mathbb{Z}
$$

y los exponentes de la constancia local $\leq 1$.

Luego establecemos:

$$
D_{N}=\underset{l \rightarrow \infty l}{\lim _{N}}
$$

y defina la topología en $D(K)$ como la topología de límite inducido, es decir:

$$
D_{N}(K)=\underset{N \rightarrow \infty N}{\lim }
$$

El fuerte espacio conjugado $D^{\prime}(K)$ se llama espacio de distribuciones de Bruhat-Schwartz.

La operación de la transformada de Fourier conserva el espacio $D(K) \circ D\left(K^{n}\right)$ (esta propiedad contrasta con el caso Arquimediano). Por lo tanto, la transformada de Fourier de una distribución definida, por dualidad, al igual que para las distribuciones de $S^{\prime}\left(R^{n}\right)$, actúa continuamente en $D^{\prime}$ $(K)$, resp. $D^{\prime}\left(K^{n}\right)$. Como en el caso de $R^{n}$, existe una teoría bien desarrollada de distribuciones sobre campos locales que incluye temas como convolución, producto directo, distribuciones homogéneas, etc. Observe en particular que una función $|x|^{\alpha-1}, R \alpha>0$, admite una continuación analítica en una distribución meromórfica.

A continuación, usaremos a menudo los subespacios de $D(K)$,

$$
\begin{gathered}
\left\langle|x|^{\alpha-1}, \varphi\right\rangle=\int_{K}|x|^{\alpha-1}[\varphi(x)-\varphi(0)] d x, \varphi \in D(K) \quad \mathfrak{R} \alpha>0 \\
\Psi\left(K^{n}\right)=\left\{\psi \in D\left(K^{n}\right) ; \psi(0)=0\right\} \\
\Phi\left(K^{n}\right)=\left\{\varphi \in D\left(K^{n}\right) ; \int_{K^{n}} \varphi(x) d^{n} x=0\right\}
\end{gathered}
$$


introducido en [1]. El espacio $\Phi\left(K^{n}\right)$ se denomina espacio de Lizorkin de las funciones de prueba del segundo tipo; es una contraparte no-arquimediano de un espacio de funciones de prueba en $R^{n}$ propuesto por Lizorkin. El espacio conjugado $\Phi^{\prime}\left(K^{n}\right)$ se llama espacio de distribuciones de Lizorkin del segundo tipo. La propiedad más importante de estos espacios es que la transformada de Fourier Fes un isomorfismo lineal de $\psi\left(K^{n}\right)$ a $\Phi\left(K^{n}\right)$, por lo tanto, también de $\Phi^{\wedge^{\prime}}\left(K^{n}\right)$ a $\psi^{\wedge^{\prime}}\left(K^{n}\right)$. Al mismo tiempo, F puede considerarse como un isomorfismo lineal de $\Phi\left(K^{n}\right)$ a $\psi\left(K^{n}\right)$.

\section{Operadores Pseudo-Diferenciales}

El operador pseudo-diferencial más simple y mejor estudiado, que actúa sobre funciones de valor complejo sobre $\mathrm{K}$, es el operador de diferenciación fraccional $D^{\alpha}, \alpha>0$, cuya investigación profunda fue iniciada por Vladimirov (2003); Se define como:

$$
\left(D^{\alpha} \varphi\right)(x)=F^{-1}\left[|\xi|^{\alpha}(F(\varphi))(\xi)\right](x), \varphi \in D(K)
$$

Tenga en cuenta que $D^{\alpha}$ no actúa en el espacio $D(K)$, ya que la función $\xi \rightarrow|\xi|^{\alpha} \mid$ No es localmente constante. Podemos afirmar, por ejemplo, que $D^{\alpha}: D(K) \rightarrow L_{2}(K)$, y la cerradura de este operador es autoadjunto en $L_{2}(K)$. Por otro lado,

$$
D^{\alpha}: \Phi(K) \rightarrow \Phi(K) \text { y } D^{\alpha}: \Phi^{\prime}(K) \rightarrow \Phi^{\prime}(K) ;
$$

Del mismo modo, para $x=\left(x_{1}, X_{2}, \ldots, X_{n}\right) \in K^{n}$, sea $\|x\|=\max _{1 \leq j \leq n}\left|x_{j}\right|$. El operador pseudo-diferencial $D^{\alpha, n}: D\left(K^{n}\right) \rightarrow L_{2}\left(K^{n}\right)$ viene dada por la expresión

$$
\left(D^{\alpha, n} \varphi\right)(x)=F^{-1}\left[\|\xi\|^{\alpha}(F(\varphi))(\xi)\right](x), \varphi \in D\left(K^{n}\right)
$$

Nosotros tenemos:

$$
D^{\alpha, n}: \Phi\left(K^{n}\right) \rightarrow \Phi\left(K^{n}\right) \text { y } D^{\alpha, n}: \Phi^{\prime}\left(K^{n}\right) \rightarrow \Phi^{\prime}\left(K^{n}\right) .
$$

Una propiedad importante de estos operadores es la posibilidad de deshacerse de la transformada de Fourier y representarlos como operadores integrales hiper-singulares. Para cualquier $u \in D(K)$,

$$
\left(D^{\alpha} u\right)(x)=\frac{1-q^{\alpha}}{1-q^{-\alpha-1}} \int_{K}|y|^{-\alpha-1}[u(x-y)-u(x)] d y
$$

La expresión en el lado derecho de (5) tiene sentido para clases más amplias de funciones, por ejemplo, para todas las funciones limitadas localmente constantes. Del mismo modo, si $u \in D\left(K^{n}\right)$, entonces:

$$
\left(D^{\alpha, n} u\right)(x)=\frac{1-q^{\alpha}}{1-q^{-\alpha-n}} \int_{K^{n}}\|y\|^{-\alpha-n}[u(x-y)-u(x)] d^{n} y
$$

Lema 1. Si u es una función localmente limitada en $K^{n}$, entonces la distribución $D^{\alpha, n} \in \Phi^{\wedge^{\prime}}\left(K^{n}\right)$ coincide con la función (6).

\section{La Transformación de Radón}

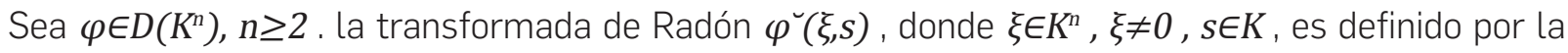
relación:

$$
\breve{\varphi}(\xi, s)=\int_{\xi . x=s} \varphi(x) d \omega_{\xi, s}(x)
$$


(ver [3]) donde $\omega_{\xi, s}$ es una medida en el hiperplano $\xi \cdot x=s$ (nosotros escribimos $\xi \cdot x=\xi_{1} \cdot x_{1}+\xi_{2} \cdot X_{2}+\cdots+\xi_{n}$. $x_{n}$ ) que para cualquier $\psi \in D\left(K^{n}\right)$,

$$
\int_{K} d s \int_{\xi \cdot x=s} \psi(x) d \omega_{\xi, s}(x)=\int_{K^{n}} \psi(x) d x
$$

La función $\varphi^{\smile}$ posee las siguientes propiedades. Es homogéneo de grado -1 en $\xi$ y $s$, es decir $\varphi$ $\smile(\sigma \xi, \sigma s)=|\sigma|^{-1} \varphi(\xi, s)$, para cualquier $\sigma \in K \mid\{0\}$.

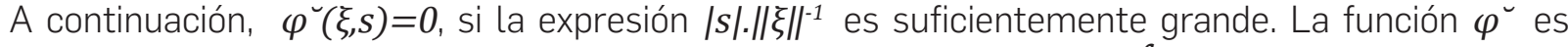

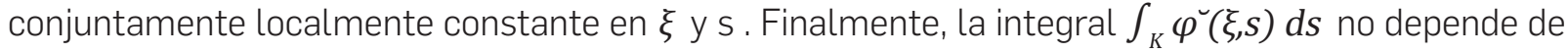
$\xi$. Tenga en cuenta que las propiedades anteriores de una función de $\xi$ y $s$ también son suficientes para que dicha función sea la transformación de radón de alguna función $\varphi \in D\left(K^{n}\right)$.

Para encontrar una conexión entre las transformaciones de Radón y Fourier (similar a la conocida para el caso de $R^{n}$, escribimos:

$$
\tilde{\varphi}(s \xi)=\int_{K^{n}} \varphi(x) \chi(s(x . \xi)) d^{n} n=\int_{K} d r \int_{\xi \cdot x=r} \varphi(x) \chi(s r) d \omega_{\xi, r}(x)=\int_{K} \chi(s r) \breve{\varphi}(\xi, r) d r
$$

y se deduce de la fórmula de inversión de Fourier que:

$$
\breve{\varphi}(\xi, r)=\int_{K} \chi(-s r) \tilde{\varphi}(s \xi) d s
$$

La fórmula de inversión para la transformación de Radón no Arquimediano es la siguiente:

$$
\varphi(x)=\frac{1-q^{n-1}}{\left(1-q^{-1}\right)\left(1-q^{-n}\right)} \int_{\|\eta\|=1}\left\langle|s|^{-n}, \breve{\varphi}(\eta, s+\eta \cdot x)\right\rangle d^{n} \eta, x \in K^{n}
$$

donde la distribución $|s|^{-n}$ se entiende en el sentido de (4). Sustituyendo (4) en (8) y comparando con (5) podemos escribir la fórmula de inversión de la siguiente forma:

$$
\varphi(x)=\frac{1}{1-q^{-1}} \int_{\|\eta\|=1}\left(D_{s}^{n-1} \breve{\varphi}(\eta, s+\eta \cdot x)\right) \mid s=0 d \eta
$$

La identidad (9) se puede probar directamente, sustituyendo (7) y calculando las integrales.

\section{Funciones Propias Radiales}

$L_{2}$-soluciones. Sea $u(x)=\psi(|x|) \in L_{2}(K)$,

$$
D^{\alpha} u=\lambda u, \lambda=q^{\alpha N}, N \in \mathbb{Z}
$$

Y u no es idénticamente cero.

Apliquemos la transformada de Fourier a ambos lados de (10).

Obtenemos:

$$
\left(|\xi|^{\alpha}-q^{\alpha N}\right) \tilde{u}(\xi)=0 \text { para todo } \xi \in K
$$

De (11) se deduce que la desigualdad $\tilde{u}(\xi) \neq 0$ solo es posible para $|\xi|=q^{N}$. Como u es una función radial, ũ también posee esta propiedad.

Por lo tanto:

$$
\tilde{u}(\xi)=\left\{\begin{array}{ll}
c & ;|\xi|=q^{N} \\
0 & ;|\xi| \neq q^{N}
\end{array} \quad, c \neq 0\right.
$$


Mediante la inversión de Fourier y la conocida fórmula de integración.

Obtenemos:

$$
u(x)=\left\{\begin{array}{ccc}
c q^{N}\left(1-q^{-1}\right) & x & ;|x| \leq q^{-N} \\
-c q^{N-1} & x & ;|x|=q^{-N+1} \\
0 & x & ;|x|>q^{-N+1}
\end{array}\right.
$$

Se puede ver fácilmente desde (12) o (13) que $u \in \Phi(K)$.

La única función propia radial $u$ con $u(0)=1$ (un análogo de la función $t \rightarrow e^{-\lambda t}, t \in R$ ) corresponde a $c=q^{-N}\left(1-q^{-1}\right)^{-1}$. Por otro lado, si $u(0)=0$, entonces $c=0$.

\section{Soluciones Generalizadas}

Consideremos la solución $u \in \Phi^{\wedge^{\prime}}(K)$ de la ecuación (10). Es natural llamar a una distribución $u \in \Phi^{\wedge^{\prime}}$ (K) radial (o esféricamente simétrica), si para algún $\omega \in K,|\omega|=1$, y cualquier $\varphi \in \Phi(K)$,

$$
\left\langle u, \varphi_{\omega}\right\rangle=\langle u, \varphi\rangle
$$

donde $\varphi_{\omega}(x)=\varphi(\omega x)$. De manera similar, definimos una distribución radial a partir de $\Psi^{\prime}(K)$. Es fácil comprobar que la transformada de Fourier mapea una distribución radial desde $\Phi^{\prime}(K)$ a una distribución radial desde $\Psi^{\prime}(K)$.

Proposición 1. Si una distribución radial $u \in \Phi^{\prime}(K)$ satisface la ecuación (10), entonces coincide, para algunos $c \in C$, con la función (13).

\section{Prueba}

Por definición de una solución generalizada, tenemos:

$$
\left\langle u, D^{\alpha} \varphi\right\rangle=\lambda\langle u, \varphi\rangle \text { para cualquier } \varphi \in \Phi(K)
$$

Se escribe $\varphi=F^{-1} \psi, \psi \in \Psi(K)$, vemos eso.

$$
\left(D^{\alpha} \varphi\right)(x)=F_{\xi \rightarrow x}^{-1}\left(|\xi|^{\alpha} \psi(\xi)\right)
$$

La función $\xi \rightarrow|\xi|^{\alpha} \psi(\xi)$ pertenece a $\Psi(K)$. Por lo tanto, considerando F Como operador de $\Phi^{\prime}(K)$ en $\Psi^{\prime}(K)$.

Nosotros podemos escribir.

$$
\left\langle u, D^{\alpha} \varphi\right\rangle=\left\langle(F u)(\xi),|\xi|^{\alpha} \psi(\xi)\right\rangle=\left\langle|\xi|^{\alpha}(F u)(\xi), \psi(\xi)\right\rangle
$$

para que lleguemos a la igualdad (11) donde esta vez $\tilde{u}=F u \in \psi^{\prime}(K)$, y la multiplicación por $|\xi|^{\alpha}-q^{\alpha N}$ se entiende en el sentido de distribución.

Por lo tanto, para cualquier $\psi \in \Psi(K)$,

$$
\left\langle(F u)(\xi),\left(|\xi|^{\alpha}-q^{\alpha N}\right) \psi(\xi)\right\rangle=0
$$

En la esfera $\left\{\xi \in K ;|\xi|=q^{l}\right\}, l \neq N$, el conjunto de funciones $\xi \rightarrow\left(|\xi|^{\alpha}-q^{\alpha N}\right) \psi(\xi)$ ejecuta el conjunto de restricciones de todas las funciones desde $\Psi(K)$. Por lo tanto, la restricción de la distribución Fu a dicha esfera es igual a cero, de modo que Fu se concentra en la esfera $S_{N}=\left\{\xi \in K ;|\xi|=q^{N}\right\}$. 
El conjunto de restricciones a $S_{N}$ de funciones de $\Psi(K)$ coincide con $D\left(S_{N}\right)=\underset{l \rightarrow \infty l_{N}}{\lim }$

donde $D_{l}\left(S_{N}\right)$ es el conjunto de funciones de valores complejos en $\mathrm{S} \_\mathrm{N}$ con los exponentes de la constancia local $\leq l$. El espacio $D_{l}\left(S_{N}\right)$ es de dimensión finita; su base puede construirse a partir de las funciones $\delta_{\sigma 0, \sigma 1, \ldots \sigma N+l-1}(\mathrm{t})\left(\sigma_{0, \sigma 1, \ldots, \sigma N+l-1} \in S, \sigma_{0} \notin P\right)$, que equivalen a 1 en los elementos $t \in S_{N}$ con las representaciones canónicas $t=\beta^{-N}\left(\sigma_{0}+\sigma_{1} \beta+\cdots+\sigma_{N+1-1} \beta^{N+l-1}+O\left(\beta^{l}\right)\right.$, y 0 en todos los demás $t \in S_{N}$.

Denotar

$$
c_{\sigma_{0}, \sigma_{1}, \ldots, \sigma_{N+l-1}}=\left\langle F u, \delta_{\sigma_{0}, \sigma_{1}, \ldots, \sigma_{N+l-1}}\right\rangle
$$

La razón de dos elementos cualquiera $=\beta^{-N}\left(\sigma_{0}+\sigma_{1} \beta+\cdots+\sigma_{N+1-1} \beta^{N+l-1}\right.$ pertenece al grupo de unidades $\cup$. La transformación de una de las funciones $\delta_{\sigma 0, \sigma 1, \ldots \sigma N+1-1}$ en otro (con el mismo $I$ ) se implementa mediante la multiplicación del argumento por la proporción adecuada. Como Fu es una distribución radial, encontramos que $c_{\sigma 0, \sigma 1, \ldots \sigma N+l-1}$ depende solo de 1 , digamos:

$$
c_{\sigma_{0}, \sigma_{1}, \ldots, \sigma_{N+l-1}}=c_{l-1}^{\prime}
$$

Al mismo tiempo,

$$
\sum_{\sigma_{N+l} \in S} \delta_{\sigma_{0}, \sigma_{1}, \ldots, \sigma_{N+l-1}, \sigma_{N+l}}=\delta_{\sigma_{0}, \sigma_{1}, \ldots \sigma_{N+l-1}}
$$

De dónde $c_{l-1}^{\prime}=q c_{1}{ }^{\prime}$ y $c_{1}{ }^{\prime}=c_{0}{ }^{\prime} q^{-1}, c_{0}{ }^{\prime} \in C$. Por lo tanto, hemos encontrado que

$$
\left\langle F u, \delta_{\sigma_{0}, \sigma_{1}, \ldots, \sigma_{N+l}}\right\rangle=c_{0}^{\prime} q^{-l}
$$

Para todo l.

Mientras tanto, el integral:

$$
\int_{|t|=q^{N}} \delta_{\sigma_{0}, \sigma_{1}, \ldots \sigma_{N+l}}(t) d t
$$

es igual a $q^{N-(N+1)-1}=q^{-1-1}$. Junto con (14), esto muestra que la restricción de la distribución Fu a la esfera $S_{N}$ es igual a una constante; fuera de $S_{N^{\prime}}$ Fu es igual a O. Por lo tanto, Fu tiene la forma (12), de modo que u coincide con la función (13).

\section{RESULTADOS}

Siguiendo un patrón clásico llamamos una función:

$$
F(t, x)=f\left(t+\omega_{1} x_{1}+\cdots+\omega_{n} x_{x}\right)
$$

$t \in K,\left(x_{1}, \ldots, X_{n}\right) \in K^{n}$, dónde $\left\|\left(\omega_{1}, \ldots, \omega_{n}\right)\right\|=1, f \in D(K)$, una onda plana no archimedeana.

\section{Proposición 2.}

Para cualquier $\alpha>0$, una onda plana no arquimediana (15) satisface la ecuación

$$
D_{t}^{\alpha} F-D_{x}^{\alpha, n} F=0
$$

\section{Prueba}

Suponga que $\mathrm{n} \geq 2$ (en el cason=1, la validez de (16) se verifica de forma directa). Calculemos $D_{t}^{\alpha, n} F$. Por la definición de $D_{t}^{\alpha, n} F$,

$$
\begin{aligned}
& \left(D_{x}^{\alpha, n} F\right)(t, x)=\frac{1-q^{\alpha}}{1-q^{-n-\alpha}} \int_{K^{n}}\left(\max _{j}\left|y_{j}\right|\right)^{-n-\alpha} \times\left[f\left(t+\sum_{j=1}^{n} \omega_{j} x_{j}-\sum_{j=1}^{n} \omega_{j} y_{j}\right)-f(t+\right. \\
& \left.\left.\sum_{j=1}^{n} \omega_{j} x_{j}\right)\right] d y_{1} \ldots d y_{n}
\end{aligned}
$$


Desde $\max _{j}\left|\omega_{j}\right|=1$, podemos elegir un índice $j_{0}$ de tal manera que $\left|\omega_{j o}\right|=1$. Supongamos para simplicidad que $\left|\omega_{1}\right|=1$. Realicemos el cambio de variables:

$$
\eta_{1}=\sum_{j=1}^{n} \omega_{j} y_{j}, \eta_{2}=y_{2}, \ldots, \eta_{n}=y_{n}
$$

Obviamente, $\max _{j}\left|\eta_{j}\right| \leq \max _{j}\left|y_{j}\right|$. Por otra parte,

$$
y_{1}=\frac{1}{\omega_{1}}\left(\eta_{1}-\omega_{2} \eta_{2}-\cdots-\omega_{n} \eta_{n}\right)
$$

de donde $\max _{j}\left|y_{j}\right| \leq \max _{j}\left|n_{j}\right|$, para que:

$$
\max _{j}\left|y_{j}\right|=\max _{j}\left|\eta_{j}\right|
$$

El jacobiano de la transformación $\left(y_{1}, \ldots, y_{n}\right) \mapsto\left(\eta_{1}, \ldots, \eta_{n}\right)$ es igual a,

y pertenece a $U$. Tenemos:

$$
\left|\begin{array}{cccc}
\omega_{1} & \omega_{2} & \cdots & \omega_{n} \\
0 & 1 & \cdots & 0 \\
\vdots & \vdots & \ddots & \vdots \\
0 & 0 & \cdots & 1
\end{array}\right|
$$

$$
\begin{aligned}
& \left(D_{x}^{\alpha, n} F\right)(t, x)=\frac{1-q^{\alpha}}{1-q^{-n-\alpha}} \int_{K^{n}}\left(\max _{j}\left|\eta_{j}\right|\right)^{-n-\alpha} \times\left[f\left(t+\sum_{j=1}^{n} \omega_{j} x_{j}-\eta_{1}\right)-f\left(t+\sum_{j=1}^{n} \omega_{j} x_{j}\right)\right] d \eta_{1} \ldots d \eta_{n} \\
& \left(D_{x}^{\alpha, n} F\right)(t, x)=\frac{1-q^{\alpha}}{1-q^{-n-\alpha}} \int_{K}\left[f\left(t+\sum_{j=1}^{n} \omega_{j} x_{j}-\eta_{1}\right)-f\left(t+\sum_{j=1}^{n} \omega_{j} x_{j}\right)\right] d \eta_{1} \times \int_{K^{n-1}}\left(\max _{1 \leq j \leq n}\left|\eta_{j}\right|\right)^{-n-\alpha} d \eta_{2} \ldots d \eta_{n}
\end{aligned}
$$

Para calcular la integral sobre $K^{n-1}$, la escribimos en la forma:

$$
\begin{gathered}
\int_{K^{n-1}}\left(\max _{1 \leq j \leq n}\left|\eta_{j}\right|\right)^{-n-\alpha} d \eta_{2} \ldots d \eta_{n}=I_{1}+I_{2} \\
I_{1}=\int_{\substack{\max \left|\eta_{j}\right|<\eta_{1} \mid \\
2<j \leq n}}\left|\eta_{1}\right|^{-n-\alpha} d \eta_{2} \ldots d \eta_{n} \quad, \quad I_{2}=\int_{\substack{\max \left|\eta_{j}\right| \geq\left|\eta_{1}\right| \\
2 \leq j \leq n}}\left(\max _{2 \leq j \leq n}\left|\eta_{j}\right|\right)^{-n-\alpha} d \eta_{2} \ldots d \eta_{n}
\end{gathered}
$$

Es bien sabido que:

$$
\int_{2 \leq j \leq n} d \eta_{2} \ldots d \eta_{n}=q^{(n-1) k}\left(1-q^{-n+1}\right)
$$

Supongamos que $\left|\eta_{1}\right|=q^{v}, v \in Z$. entonces:

$$
\begin{gathered}
I_{1}=\left|\eta_{1}\right|^{-n-\alpha} \sum_{k=-\infty}^{v-1} q^{(n-1) k}\left(1-q^{-n+1}\right)=\left|\eta_{1}\right|^{-n-\alpha} q^{(n-1)(v-1)}=q^{-(n-1)}\left|\eta_{1}\right|^{-1-\alpha} \\
I_{2}=\sum_{k=v}^{\infty} q^{-k(n+\alpha)} q^{(n-1) k}\left(1-q^{-n+1}\right)=\left(1-q^{-n+1}\right) \sum_{k=v}^{\infty} q^{-k(1+\alpha)}=\frac{1-q^{-n+1}}{1-q^{-\alpha-1}}\left|\eta_{1}\right|^{-1-\alpha}
\end{gathered}
$$


así que eso,

$$
\int_{K^{n-1}}\left(\max _{1 \leq j \leq n}\left|\eta_{j}\right|\right)^{-n-\alpha} d \eta_{2} \ldots d \eta_{n}=\frac{1-q^{-n-\alpha}}{1-q^{-\alpha-1}}\left|\eta_{1}\right|^{-\alpha-1}
$$

Por lo tanto:

$$
\begin{aligned}
\left(D_{x}^{\alpha, n} F\right)(t, x) & =\frac{1-q^{\alpha}}{1-q^{-\alpha-1}} \int_{K}\left|\eta_{1}\right|^{-1-\alpha}\left[f\left(t+\sum_{j=1}^{n} \omega_{j} x_{j}-\eta_{1}\right)-f\left(t+\sum_{j=1}^{n} \omega_{j} x_{j}\right)\right] d \eta_{1} \\
& =\left(D_{t}^{\alpha} F\right)(t, x)
\end{aligned}
$$

lo que significa que F satisface la ecuación (16).

\section{Problemas de Cauchy}

Deje $\varphi \in D\left(K^{n}\right)$ Buscaremos una solución $F(t, x)$ de la ecuación (16) que satisfaga la condición inicial:

$$
F(0, x)=\varphi(x), x \in K^{n}
$$

o la condición inicial modificada:

$$
\left(D_{t}^{n-1} F\right)(0, x)=\varphi(x), x \in K^{n}
$$

Por supuesto, las condiciones (17) y (18) coinciden si $\mathrm{n}=1$.

Deje $\varphi(\xi, s)$ ser la transformación de radón de la función inicial $\varphi$.

Denotar

$$
\Gamma(t, x, u)=\breve{\varphi}(u, t+u \cdot x), t \in K, x, u \in K^{n},\|u\|=1 .
$$

Consideremos las funciones.

$$
\begin{gathered}
F_{1}(t, x)=\left(1-q^{-1}\right)^{-1} \int_{\|u\|=1}\left(D_{t}^{n-1} \Gamma\right)(t, x, u) d^{n} u \\
F_{2}(t, x)=\left(1-q^{-1}\right)^{-1} \int_{\|u\|=1} \Gamma(t, x, u) d^{n} u
\end{gathered}
$$

\section{Teorema 1.}

Las funciones $F_{1}(t, x)$ y $F_{2}(t, x)$ son radiales en $t$, conjuntamente localmente constantes en $(t, x)$, soluciones limitadas del problema de Cauchy (16), (17) y el Cauchy modificado problema (16), (18) respectivamente.

\section{Prueba}

De la identidad (7) se deduce que $\breve{\varphi}(\xi, r)$ pertenece a $D(K)$ en $r$ uniformemente con respecto $\xi \in K^{n}$, $\|\xi\|=1$ - existe un conjunto compacto en $K$, fuera del cual $\breve{\varphi}(\xi, \cdot)$ Desaparece, para todo lo anterior $\xi, y \breve{\varphi}(\xi, r+r)=\breve{\varphi}(\xi, r)$ si $\left|r^{\prime}\right| \leq q^{-1}$ donde $l$ no depende $\xi$. Esto significa que $\Gamma$ es localmente constante en t,x , uniformemente con respecto a $u \in K^{n}$ con $\|u\|=1$. Además, $\Gamma$ y $D_{t}^{n-1} \Gamma$ están delimitados, uniformemente con respecto a $u$. Estas propiedades permiten cambiar el orden de integración mientras se calculan $D_{t}^{\alpha} F_{j}$ y $D_{x}^{\alpha, n}$ Luego, la Proposición 2 muestra que $F_{1}$ y $F_{2}$ satisfacen la ecuación (16). Las condiciones iniciales se cumplen debido a la fórmula de inversión de radón (9).

Para verificar que $F_{2}(t, x)$ es radial en $t$, notamos que

$$
\breve{\varphi}(\omega \xi, \omega s)=\breve{\varphi}(\xi, s),|\omega|=1
$$


en virtud de la propiedad de homogeneidad de $\breve{\varphi}$. Por lo tanto

$$
\Gamma(\omega t, x, u)=\breve{\varphi}(u, \omega t+u \cdot x)=\breve{\varphi}\left(\omega^{-1} u, t+\left(\omega^{-1} u\right) \cdot x\right)=\Gamma\left(t, x, \omega^{-1} u\right),
$$

así que eso

$$
F_{2}(\omega t, x)=\left(1-q^{-1}\right)^{-1} \int_{\|u\|=1} \Gamma\left(t, x, \omega^{-1} u\right) d^{n} u=F_{2}(t, x)
$$

Dado que el operador $D_{t}^{n-1}$ conmuta con el operador $f(t) \mapsto f(\omega t),|\omega|=1$, encontramos también que $F_{1}$ es radial en $t$.

Estudiemos la solución $F_{2}(t, x)$ del problema Cauchy modificado (16), (18) con un poco más de detalle. Usando la conexión (7) entre las transformadas de Fourier y Radón obtenemos que:

$$
\int_{\|u\|=1} \Gamma(t, x, u) d^{n} u=\int_{K} \chi(-s t) d s=\int_{\|u\|=1} \chi(-s(u \cdot x)) \tilde{\varphi}(s u) d^{n} u
$$

Próximo,

$$
\int_{\|u\|=1} \chi(-s(u \cdot x)) \tilde{\varphi}(s u) d^{n} u=\int_{K^{n}} \varphi(y) d^{n} y \int_{\|u\|=1} \chi(s(u \cdot(y-x))) d^{n} u
$$

Mediante la conocida fórmula de integración (ver, por ejemplo, [12]),

$$
\int_{\|u\|=1} \chi(s(u \cdot(y-x))) d^{n} u=\left\{\begin{array}{cl}
1-q^{-n} ;|s| .\|y-x\| \leq 1 \\
-q^{-n} & ;|s| .\|y-x\|=q \\
0 & ;|s| .\|y-x\|>q
\end{array}\right.
$$

así que eso;

$$
\begin{aligned}
& \int_{\|u\|=1} \chi(-s(u \cdot x)) \tilde{\varphi}(s u) d^{n} u=\left(1-q^{-n}\right) \int_{\|y-x\| \leq|s|^{-1}} \varphi(y) d^{n} y- \\
& q^{-n} \int_{\|y-x\|=q|s|^{-1}} \varphi(y) d^{n} y
\end{aligned}
$$

\section{Proposición 3.}

Suponga que

$$
\begin{aligned}
& \varphi(x)=0 \text { para }\|x\|>q^{N}, \varphi(y)=\varphi(x) \text { si }\|y-x\| \leq q^{-v}, v, N \in \mathbb{N} . \text { Entonces } \\
& F_{2}\left(t+t^{\prime}, x\right)=F_{2}(t, x) \text {, si }\left|t^{\prime}\right| \leq q^{-v}, y F_{2}(t, x)=0 \text { para }|t|>q^{N+1} .
\end{aligned}
$$

\section{Prueba}

Por (19) y (20),

$$
F_{2}(t, x)=\left(1-q^{-1}\right)^{-1} \int_{K} \chi(-s t) R(s, t) d s
$$

Donde:

$$
R(s, x)=\left(1-q^{-n}\right) \int_{\|y-x \leq\| \leq|s|^{-1}} \varphi(y) d^{n} y-q^{-n} \int_{\|y-x\|=q|s|^{-1}} \varphi(y) d^{n} y
$$

Si $|s| \geq q^{v+1}$, entonces:

$$
\begin{aligned}
R(s, x) & =\varphi(x)\left\{\left(1-q^{-n}\right) \int_{\|y\| \leq|s|^{-1}} d^{n} y-q^{-n} \int_{\|y-x\|=q|s|^{-1}} d^{n} y\right\} \\
& =\varphi(x)|s|^{-n}\left[\left(1-q^{-n}\right)-q^{-n} \cdot q^{n}\left(1-q^{-n}\right)\right]=0
\end{aligned}
$$

así que eso:

$$
F_{2}(t, x)=\left(1-q^{-1}\right)^{-1} \int_{|s| \leq q^{v}} \chi(-s t) R(s, x) d s
$$


lo que implica la constancia local requerida en $t$.

sea $|t|>q^{N+1}$. Entonces existe tal elemento $s_{0} \in K,\left|s_{0}\right|=q^{-N-1}$, que $\chi\left(s_{0} t\right) \neq 1$. Si $\|x\| \leq q^{N}$, entonces $\varphi(y)=0$ para $\|y-x\|>q^{N}$. Por lo tanto, para $|s|<q^{-N}$ el segundo sumando en el lado derecho de (22) es igual a cero, mientras que el dominio de integración en el primer sumando puede fijarse como $\left\{y \in K^{n} ;\|y-x\| \leq q^{N}\right\}$, si $|s|<q^{-N}$. Por lo tanto, $R(s, x)$ es constante en $s$ en el conjunto $\left\{s \in K ;|s|<q^{-N}\right\}$, lo que implica la igualdad $R\left(s+s_{0}, x\right)=R(s, x)$ para todos los valores de $s$. Haciendo en (21) el cambio de variables $s=s^{\prime}+s_{0}$ llegamos a la identidad $F_{2}(t, x)=\chi\left(s_{0} t\right) F_{2}(t, x)$, que produce la igualdad requerida $F_{2}(t, x)=0$.

Tenga en cuenta que la constancia local de $F_{2}$ en t puede interpretarse como una contrapartida del dominio finito de dependencia para una ecuación de onda clásica: si la función inicial $\varphi$ es tal que $\varphi(x)=0$ fuera de un conjunto compacto $C \subset K^{n}$, luego $F_{2}(t, x)=0$ para $x \in K^{n} \backslash C$, al menos en algún entorno del origen $t=0$. Mientras tanto, el hecho de que $F_{2}(t, x)$ desaparece, como $|t|$ se vuelve lo suficientemente grande (para un $\|x\|$ dado), se asemeja al principio de Huygens, la existencia del borde posterior de una onda.

\section{Teorema de Unicidad}

Aquí consideramos el problema de singularidad en la clase de soluciones generalizadas, radiales en t.

Denote con $\Phi^{\prime}\left(K, \Phi^{\prime}\left(K^{n}\right)\right)$ el conjunto de distribuciones sobre el espacio de la función de prueba $\Phi(K)$, con valores en $\Phi^{\prime}\left(K^{n}\right)$.

Teorema 2. Sea $F \in \Phi^{\prime}\left(K, \Phi^{\prime}\left(K^{n}\right)\right)$ una solución generalizada de la ecuación (16), es decir

$$
\left\langle\left\langle F, D_{t}^{\alpha} \varphi_{1}\right\rangle, \varphi_{2}\right\rangle=\left\langle\left\langle F, \varphi_{1}\right\rangle, D_{x}^{\alpha, n} \varphi_{2}\right\rangle
$$

para cualquier $\varphi_{1} \in \Phi(K), \varphi_{2} \in \Phi\left(K^{n}\right)$. Si $F$ es radial en $t$, entonces $F \in D\left(K, \Phi^{\prime}\left(K^{n}\right)\right)$. Si, además, $F(0, x)=0 \circ\left(D_{t}^{n-1} F\right)(0, x)=0$, entonces $F(t, x) \equiv 0$.

\section{Prueba}

Denote por e $\tilde{F}(t, \cdot)$ la transformada de Fourier de $F$ en la variable $x$; como de costumbre, abusamos un poco de la notación, escribiendo una distribución en la variable t en función de t. Para cualquier $\psi \in \Psi\left(K^{n}\right)$ tenemos.

$$
D_{t}^{\alpha}\langle\tilde{F}(t, \cdot), \psi\rangle=\left\langle\|\xi\|^{\alpha} \tilde{F}(t, \xi), \psi(\xi)\right\rangle
$$

Si $\operatorname{supp} \psi \subset S_{N}=\left\{\xi \in K^{n} ;\|\xi\|=q^{N}\right\}, N \in \mathbb{N}$, entonces

$$
D_{t}^{\alpha}\langle\tilde{F}(t, \cdot), \psi\rangle=q^{\alpha N}\langle\tilde{F}(t, \cdot), \psi\rangle
$$

Según la Proposición 1 , la función $\langle\tilde{F}(t, \cdot), \psi\rangle$ tiene la forma (13) con $t$ sustituida por $x$ y alguna $c \in C$ . Si $\psi \in \Psi\left(K^{n}\right)$, entonces $\psi$ es una suma de un número finito de funciones soportadas en esferas $S_{N}$. Tomando, en particular, $\psi=\tilde{\varphi}, \varphi \in \Phi\left(K^{n}\right)$, encontramos que $\langle F(t, \cdot), \varphi\rangle$ pertenece a $D(K)$ en la variable t, para cualquier $\varphi \in \Phi\left(K^{n}\right)$.

Si $F(0, \cdot)$ entonces también $F(0, \cdot)=0$. Si $\psi \in \Psi\left(K^{n}\right)$, supp $\psi \subset S_{N}$ entonces, como hemos visto, $\left\langle F^{*}(t, \cdot), \psi\right\rangle$ , tiene la forma (13), y la suposición e $F^{\sim}(0, \cdot)=0$ implica la igualdad $\mathrm{c}=0$, de donde $\langle\tilde{F}(t, \cdot), \psi\rangle=0, y$ $\tilde{F}(t, \cdot)=0$ (ya que $\psi$ y $N$ son arbitrarios) y $F(t, \cdot)=0$.

Luego, si una función $u(t)$ tiene una forma (13), entonces su transformada de Fourier tiene una forma (12), y es fácil de encontrar $\left(D^{n-1} u\right)(t)$ :

$$
\left(D^{n-1} u\right)(t)=\left\{\begin{array}{cl}
c\left(1-q^{-1}\right) q^{N n} & ;|t| \leq q^{-N} \\
-c q^{N n-1} & ;|t|=q^{-N+1} \\
0 & ;|t|>q^{-N+1}
\end{array}\right.
$$


Repitiendo los argumentos anteriores, encontramos que la igualdad $\left(D^{n-1} F\right)(0, x)$ implica $F(t, x) \equiv 0$.

Se deduce del Lema 1, que las soluciones localmente limitadas de la ecuación (16) son soluciones generalizadas de la clase considerada en el Teorema 2. Por lo tanto, las soluciones de los problemas de Cauchy construidas en el Teorema 1 son únicas en la clase de radial en t, limitadas localmente funciones constantes Es natural ver soluciones como las soluciones clásicas de la ecuación de onda no-arquimediano (16).

\section{DISCUSIÓN}

En la presente investigación, se evidencia la existencia y unicidad de la ecuación de la onda plana sobre un espacio no-arquimediano con amortecimiento, a través de las pseudo diferenciales y las funciones de prueba, Albeverio et al. (2006), resolvieron la ecuación de la onda sobre un espacio p-adico, que es un ejemplo de un espacio no-arquimediano.

\section{CONCLUSIONES}

- El empleo de pseudo diferencial en espacio no arquimediano, funciones de prueba y la trasformada de fourrier en un espacio no arquimediano nos garantiza la existencia y unicidad de solución de la ecuación de la onda plana sobre un espacio no-arquimediano.

\section{REFERENCIAS BIBLIOGRÁFICAS}

Albeverio, S., Khrennikov, A. \& Shelkovich, V. (2003). Associated homogeneous p-adic distributions. Journal of Mathematics Analysis and Applications, 313(1), 64-83. DOl:10.1016/j. jmaa.2005.05.016

Chernov, V. (1970). Homogeneous distributions and the Radon transform in the space of rectangular matrices over a continuous locally compact disconnected field. Soviet Math. Dokl, 415-418.

Cruz, H. (2018). Aplicación del Teorema de Hahn Banach No-Arquimediano: Una introducción a los Espacio Vectorial Normado No-Arquimediano. Epaña: Editorial Académica Española.

Eidelman, S., Ivasyshen, S. , \& Kochubei, A. (2004). Analytic Methods in the Theory of Differential and Pseudo-Differential Equations of Parabolic Type. basel: Birkh"auser.

Gelfand, I., Graev, M. \& Piatetski-Shapiro, I. (1969). Representation Theory and Automorphic. Philadelphia: Saunders.

Helgason, S. (1980). The Radon Transform. boston: Brikhäuser.

Kochubei, A. (2001). Pseudo-Differential Equations and Stochastics over Non-Archimedean Fields. New York: Marcel Dekker.

Kochubei, A. (2009). On a p-adic waver equation. Proceedings of the Steklov Institute of Mathematics, 143-147. DOI:10.1134/S0081543809020138

Kozyrev, S. (2004). p-Adic pseudo-differential operators: methods and applications. Proceedings of the Steklov Institute of Mathematics, 138(3), 143-153. DOI: https://doi.org/10.4213/tmf31

Samko, S. (2001). Hypersingular Integrals and Their Applications. London: Taylor and Francis.

Schikhof, W. (1984). Ultrametric Calculus An Introduction to p-adic análisis. Cambrigde University Pres. 
Schikhof, W. (2003). A Crash Course In p-Adic Analysis. Cambrigde University Pres.

Schneide, P. (2005). Nonarchimedean Fuctional Analysis. New York: Berlin-Heidel-Berg.

Taibleson, M. (1968). Harmonic analysis on n-dimensional vector spaces over local fields. I. Basic results on fractional integration. Mathematische Annalen, 176, 191-207. http://eudml.org/ doc/161690

Vladimirov, V. (2003). Tables of Integrals of Complex-Valued Functions of p-Adic Arguments. Moscow: Steklov Mathematical Institute, 284(2), 3 - 88. DOl: https://doi.org/10.4213/spm5

Zuñiga, G. (2004). Pseudo-differential equations connected with p-adic forms and local zeta functions. Bulletin of the Australian Mathematical Society, 70(1), 73-86. DOl: https://doi.org/10.1017/S0004972700035838 